\title{
Minnelide effectively eliminates CD133 side population in pancreatic cancer
}

\author{
Alice Nomura', Olivia McGinn', Vikas Dudeja', Veena Sangwan', Ashok K. Saluja ${ }^{1,2}$ and Sulagna Banerjee ${ }^{1,2^{*}}$
}

\begin{abstract}
Background: Pancreatic Ductal Adenocarcinoma (PDAC) is a devastating disease hallmarked by limited patient survival. Resistance to chemotherapy, a major cause of treatment failure in PDAC patients, is often attributed to Cancer Stem Cells (CSCs). Pancreatic CSCs are a small subset of quiescent cells within a tumor represented by surface markers like CD133. These cells are responsible not only for tumor recurrence, but also poor prognosis based on their "stem-like" characteristics. At present, conventional therapy is directed towards rapidly dividing PDAC cells and thus fails to target the CSC population.

Methods: MIA PaCa-2, S2-013 and AsPC-1 were treated with $12.5 \mathrm{nM}$ triptolide (12 T cells) for 7 days. The surviving cells were recovered briefly in drug-free growth media and then transferred to Cancer Stem cell Media (CSM). As a control, untreated cells were also transferred to CSM media (CSM). The $12 \mathrm{~T}$ and CSM cells were tested for stemness properties using RNA and protein markers. Low numbers of CSM and 12 T cells were implanted subcutaneously in athymic nude mice to study their tumorigenic potential. 12 T and CSM cells were sorted for CD133 expression and assayed for their colony forming ability and sphere forming ability. Invasiveness of 12 T cells, CSM and MIA PaCa-2 were compared using Boyden chamber assays.

Results: Treated $12 \mathrm{~T}$ cells displayed increased expression of the surface marker CD133 and the drug transporter ABCG2 compared to untreated cells (CSM cells). Both 12 T and CSM cells formed subcutaneous tumors in mice confirming their tumor-initiating properties. When tested for invasion, $12 \mathrm{~T}$ cells had increased invasiveness compared to CSM cells. CD133 ${ }^{+}$cells in both CSM and $12 \mathrm{~T}$ showed greater colony and sphere forming ability compared to CD133- cells from each group. Consistent with these data, when injected subcutaneously in mice, CD133- cells from CSM or $12 \mathrm{~T}$ did not form any tumors whereas CD133 ${ }^{+}$cells from both groups showed tumor formation at a very low cell number. Despite pre-exposure to triptolide in $12 \mathrm{~T} \mathrm{CD} 133^{+}$cells, treatment of tumors formed by these cells with Minnelide, a triptolide pro-drug, showed significant tumor regression.
\end{abstract}

Conclusion: Our results indicated that triptolide enhanced and enriched the "stemness" in the PDAC cell lines at a low dose of $12.5 \mathrm{nM}$, but also resulted in the regression of tumors derived from these cells.

\section{Background}

Pancreatic cancer is one of the most aggressive malignancies with an extremely poor survival rate [1]. Even for patients who undergo potentially curative resection, the 5 -year survival is less than $5 \%$ due to local recurrence and metastasis $[2,3]$. Many different chemotherapeutic agents, including the current standard of care, Gemcitabine, have failed to demonstrate any significant

\footnotetext{
* Correspondence: sbanerje@umn.edu

${ }^{1}$ Department of Surgery, Division of Basic and Translational Research, University of Minnesota, Minneapolis, MN 55455, USA

${ }^{2}$ Masonic Cancer Center, University of Minnesota, Minneapolis, MN, USA
}

survival advantage in patients with pancreatic adenocarcinoma. Emerging evidence has shown that cancer stem cells (CSCs), a small subset of quiescent cells within a tumor, are responsible for tumor recurrence [4].

The significance of CSCs in hematological malignancy as well as in solid cancers is well known [5]. Pancreatic cancer stem cells (PCSC) were identified in 2007, when several groups demonstrated the presence of CD24, CD44, epithelial specific antigen (ESA) triple positive markers or CD133 positive cells had the ability to initiate tumor formation in animals at very low numbers $[6,7]$. Since then, many such surface markers have 
been identified [8-10]. These tumor initiating cells (TICs) or CSCs are thought to be responsible for not only tumor recurrence but also chemo-resistance and metastatic spread of a tumor. Pancreatic cancer stem cells have been reported to be resistant to gemcitabine induced apoptosis [7]. Later, Shah et al [11] and Du et al [12] established gemcitabine-resistant pancreatic cancer cell lines and found that resistant cells comprised of more cells with cancer stem cell-like phenotypes compared to the parental cells.

Expression of the TIC marker CD133 in several cancers is shown to be associated with increased expression of drug transporters like ABCG2 [13, 14]. Similarly, treatment with low concentrations of a chemotherapeutic agent like gemcitabine has been reported to enrich for CSC-like properties in a number of cancers $[11,15,16]$. Chemo-resistant CSCs in a tumor can be characterized by "Side Population (SP) analysis" [17]. SP cells can rapidly efflux lipophilic fluorescent dyes to produce a characteristic profile based on fluorescence-activated flow cytometric analysis $[18,19]$. Although representing only a small fraction of the whole cell population, they appear to be enriched in stem-like cells that can initiate tumors. Thus, they could provide a useful tool and a readily accessible source for cancer stem cell studies [20,21]. SP cells identified in bone marrow obtained from patients with acute myeloid leukemia (AML) are candidate leukemic stem cells [22, 23]. They have also been identified in various human solid tumors and cancer cell lines [12, 24]. However, the reports of their presence within pancreatic tumor have been rare.

Apart from imparting chemo-resistance to tumors, cancer stem cells and SP cells are also associated with increased invasiveness [24]. CD133 expression, for example, has been correlated with increased metastasis and poor prognosis in a number of cancers like colorectal cancer and hepatocellular carcinoma $[25,26]$. In pancreatic cancer, $\mathrm{CD} 133^{+} \mathrm{CXCR}^{+}$population and $\mathrm{CD} 44^{+} \mathrm{Met}^{+}$population show increased metastatic potential when compared to the respective negative population [7, 27]. Consistent with this, genes involved in epithelial-mesenchymaltransition (EMT) are overexpressed in cancer stem cells resulting in their increased invasiveness [28, 29]. Further, recent evidence has demonstrated the surface expression of CD133 induces this invasive phenotype through the activation of NF-kB signaling [14].

Triptolide, a diterpene triepoxide, and its water-soluble prodrug, Minnelide, have been very effective in a number of preclinical cancer models, including pancreatic cancer murine models [30-32]. Recently, we demonstrated that Minnelide is effective in not only reducing the bulk tumor, but also in targeting the tumor initiating $\mathrm{CD}_{133^{+}}$population in pancreatic cancer [33]. However, the effect of triptolide on drug resistant side-population has not been tested.
In the current study, we have developed a model in which treatment with very low dose of triptolide of pancreatic cancer cell lines MIA PaCa-2, S2-013 and AsPC1 results in a "drug-tolerant" population of cells that are enriched for most "stemness" or tumor initiating cell (TIC) markers for pancreatic cancer. These cells demonstrated increased DNA dye efflux and formed tumors at low cell concentration in athymic nude mice. Furthermore, these cells showed increased invasive properties and increased expression of EMT genes. Interestingly, when treated with Minnelide, these tumors showed complete regression, indicating that Minnelide can effectively target pancreatic cancer stem like cells.

\section{Results}

Long-term triptolide treatment enriches for SP cells

To determine if exposure to a very low dose of triptolide was effective in enriching for the chemo-resistant side population in pancreatic cancer cell lines, we treated pancreatic cancer cell lines MIA PaCa-2, S2-013 and AsPC-1 with $12.5 \mathrm{nM}$ triptolide for 7 days. At the end of this study, approximately $20 \%$ of cells were viable (Fig. 1a). These cells (12 T) showed increased expression of the drug transporter gene ABCG2 (Fig. 1b) compared to the control cells (CSM). To evaluate if expression of drug transporter genes was consistent with the DNA dye efflux property of these cells, we measured the ability of $12 \mathrm{~T}$ cells to efflux the DNA dye Hoechst 33342. As expected, $12 \mathrm{~T}$ cells showed increased DNA dye efflux property compared to the CSM cells and were thus similar to the "side-population" or SP cells (Fig. 1c and d).

\section{Side population cells are enriched for CSC-like properties}

SP cells generated as described in the previous section were assayed for previously identified pancreatic cancer stem cell markers. 12 T cells from all cells lines showed enrichment for CD133 (Fig. 2a) and CD24 ${ }^{+} \mathrm{CD} 44^{+} \mathrm{ESA}^{+}$ populations (Fig. 2b) compared to parental cell lines (MIA PaCa-2, S2-013 and AsPC-1). Additionally, $12 \mathrm{~T}$ cells also showed increased expression of the stemness genes Sox2, Oct4 and Nanog as well as several developmental genes known to be deregulated in cancer stem cells, such as SHH, Notch1, Gli1, and Jagged gene expression (Additional file 1: Table S1) and protein expression (Fig. 2c).

Since the definitive test of stemness is their ability to form tumors in animals, we injected 1000 and 10,000 MIA PaCa-2, CSM-MIA and 12 T-MIA cells in the flanks of athymic nude mice. Mice with MIA PaCa-2 cells did not form tumors until the end of the study $(n=5)$, whereas mice with CSM cells showed a tumor take of $11 \%$ ( 1 of 9 formed tumors) in the 1000 cell group and $22 \%$ (2 of 9 formed tumors) in the 10,000 cell group (Fig. 2d, e). Maximum tumorigenicity was seen in the 


\section{A}

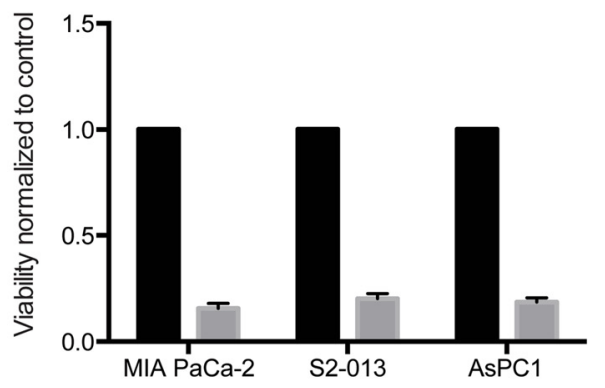

B
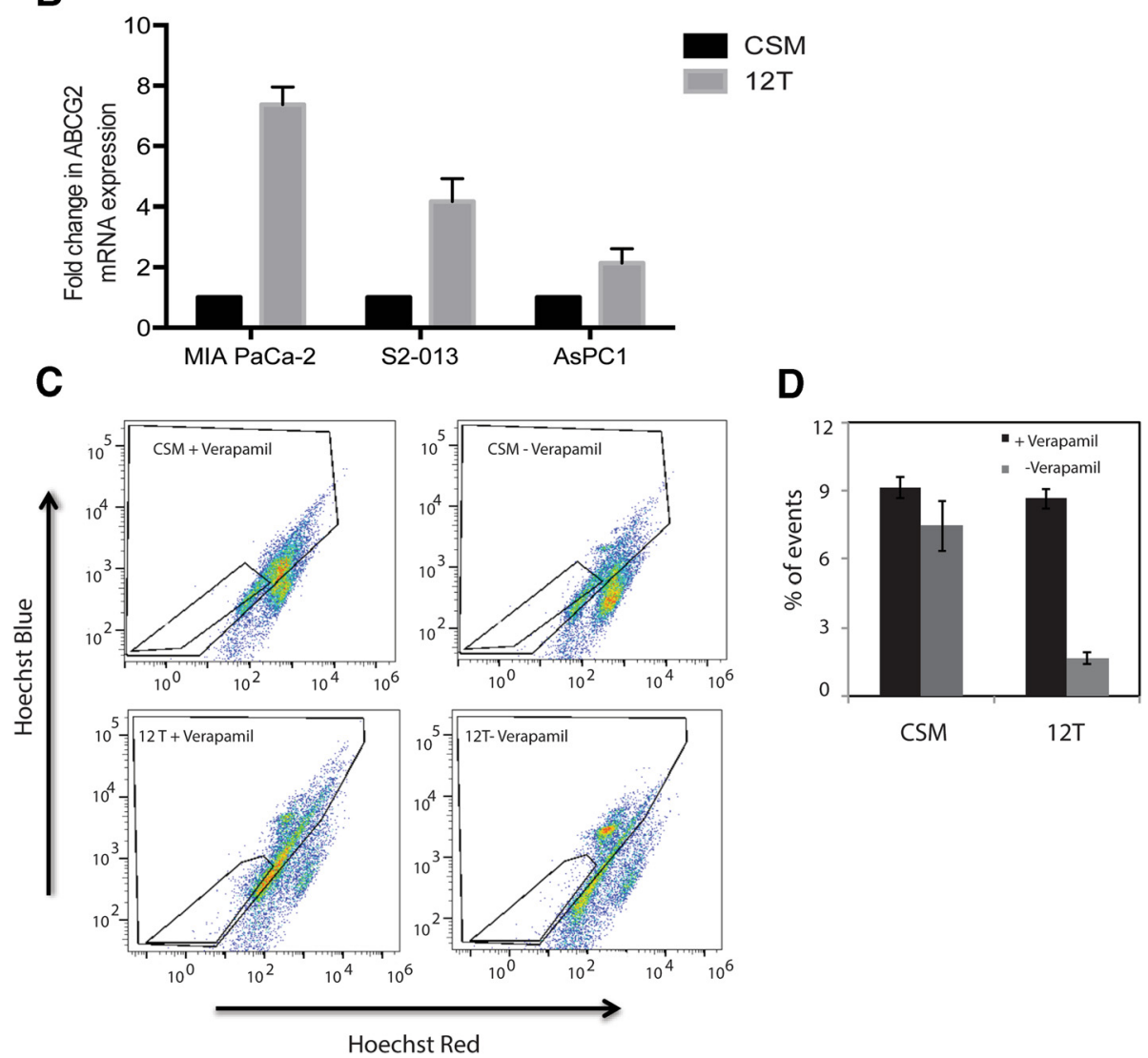

Untreated

12.5 nM Triptolide (Day 6)

2T

Fig. 1 a Treatment of pancreatic cancer cell lines MIA PaCa-2, S2-013 and AsPC1 with 12.5 nM triptolide for 7 days resulted in $20 \%$ viable cells. with increased DNA dye efflux in MIA PaCa-2 12 T cells. d Quantitation of inhibition of DNA dye efflux on treatment with Verapamil, an inhibitor of $\mathrm{ABC}$ transporter in $12 \mathrm{~T}$ and CSM cells. The error bars represent SEM $\left(n=4 ;{ }^{*}=p<0.05\right)$

$12 \mathrm{~T}$ group where mice receiving 1000 cells showed a $44 \%$ tumor take (4 of 9 mice formed tumors) and mice receiving 10,000 cells showed $87 \%$ tumor take (7 of 8 mice formed tumors) (Fig. 2d, e; summarized in Additional file 2: Table S2).

Triptolide enriched $\mathrm{CD} 133^{+}$cells show increased stemness and tumor initiation properties

Since treatment with low concentration of triptolide enriched for $\mathrm{CD} 133^{+}$cells in pancreatic cancer cell lines, we next sorted for $\mathrm{CD} 133^{+}$cells and evaluated stemness properties using the classical colony forming and sphere forming assays in vitro and tumor forming activity in vivo.

Sorted CD $133^{+}$cells from both CSM and $12 \mathrm{~T}$ formed colonies at high cell dilutions (100 cells) where as CD133- population did not (Fig. 3a). Similarly, sphere formation was observed in $\mathrm{CD}_{133^{+}}$population in both CSM and $12 \mathrm{~T}$ where as CD133- ${ }^{-}$population from either group did not (Fig. 3b). To study if $\mathrm{CD}_{133^{+}}$population 

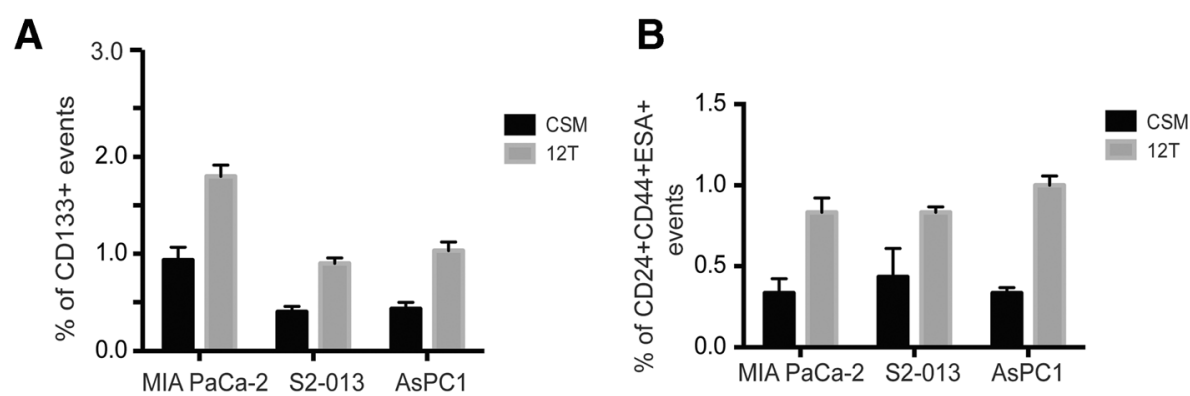

\section{C}

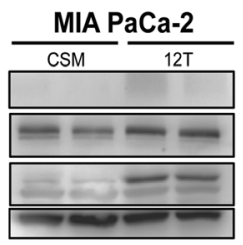

D

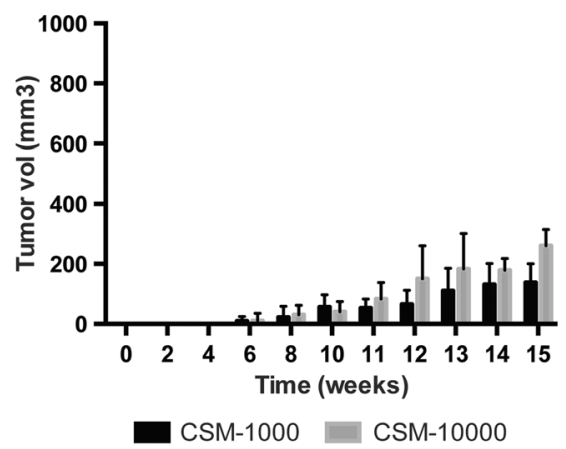

E

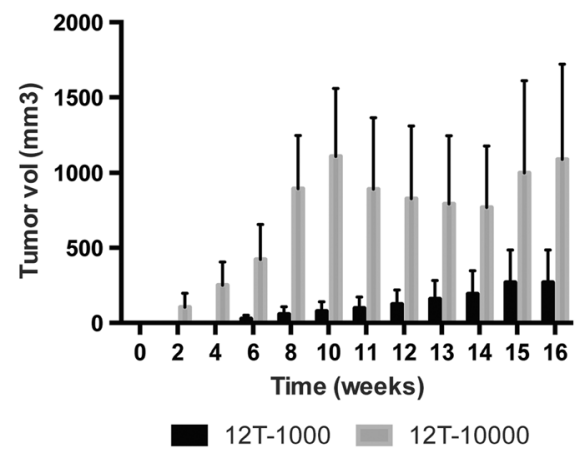

Fig. 2 a 12 T cells showed higher percentage of CD133+ cells in all three cell lines tested: MIA PaCa-2, S2-013, AsPC1. b 12 T cells also showed increased $\mathrm{CD}_{24}{ }^{+} \mathrm{CD} 44^{+} \mathrm{ESA}{ }^{+}$cells compared to CSM cells. CSM and $12 \mathrm{~T}$ cells showed increased expression of stemness proteins like Jagged, Notch1 and Gli1 (c) Tumor progression in athymic nude mice from (d) CSM cells and (e) 12 T cells. The error bars represent SEM $\left(n=4\right.$; $\left.{ }^{*}=p<0.05\right)$

was tumorigenic, we sorted cells based on CD133 expression and implanted them subcutaneously in the flanks of athymic nude mice. Consistent with the in vitro surrogate experiments, $\mathrm{CD}_{133^{+}}$cells from both CSM and $12 \mathrm{~T}$ groups formed tumors in mice where as CD133- cells did not (Fig. 3c, d). The tumorigenicity of the $12 \mathrm{~T}$ and the CSM cells is tabulated in Additional file 3: Table S3. The H\&E staining of the tumors obtained from CSM or $12 \mathrm{~T}$ cells did not show any major difference in histology however increased ALDH1 staining was observed in tumors derived from 12 T cells (Fig. 3e).

Triptolide enriched cells show increased invasiveness and increased expression of EMT genes

Increased CD133 expression has been correlated with increased invasiveness [14, 33]. To test this, we compared CSM cells with $12 \mathrm{~T}$ cells for their invasiveness by a Boyden chamber invasion assay (Fig. 4a). $12 \mathrm{~T}$ cells were 3 times more invasive compared to the CSM cells. To confirm this further, we looked for expression of
EMT genes using a PCR array. Consistent with the invasion assay, the $12 \mathrm{~T}$ cells showed increased expression of a number of genes involved in EMT. Transcription factors regulating EMT (SNAI1, SNAI2, SOX10, TWIST1, ZEB1, ZEB2) were overexpressed in $12 \mathrm{~T}$ genes when compared with the CSM cells (Fig. 4b). EMT is also associated with a re-organization of cytoskeletal proteins. Classically, expression of $\mathrm{CDH} 1$ is downregulated at the onset of EMT and the expression of $\mathrm{CDH} 2$ is increased. In keeping with this, we found that $\mathrm{CDH} 1$ was downregulated in $12 \mathrm{~T}$ cells while $\mathrm{CDH} 2$ was upregulated. In agreement with our data showing increased invasiveness of $12 \mathrm{~T}$ cells compared to controls, increase in the matrix metalloproteases (MMP2/3/9) involved in extracellular matrix reorganization of the tumor microenvironment during EMT were found to be upregulated in $12 \mathrm{~T}$ cells (Fig. 4c). To confirm these further, we looked for expression of classical EMT markers like Vimentin in the tumors developed from $12 \mathrm{~T}$ and CSM cells. As seen in vitro, the tumor cells developed from $12 \mathrm{~T}$ 
A

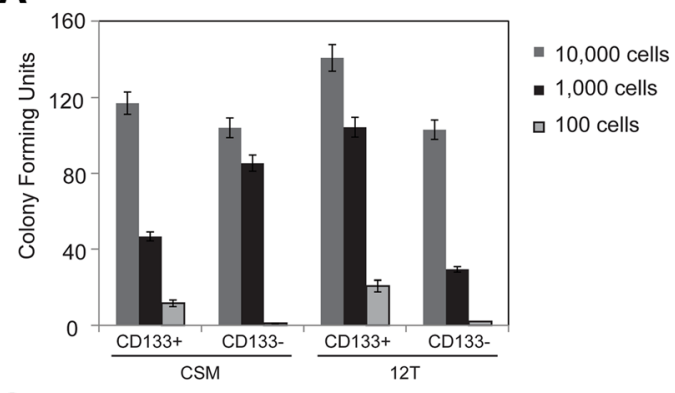

C

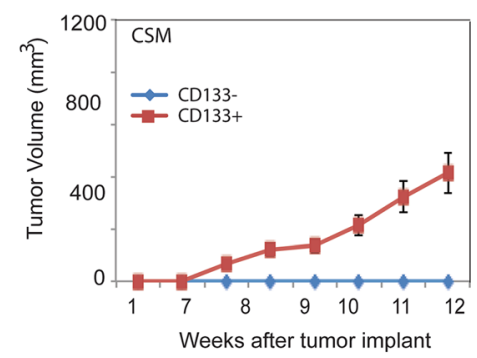

$\mathbf{E}$
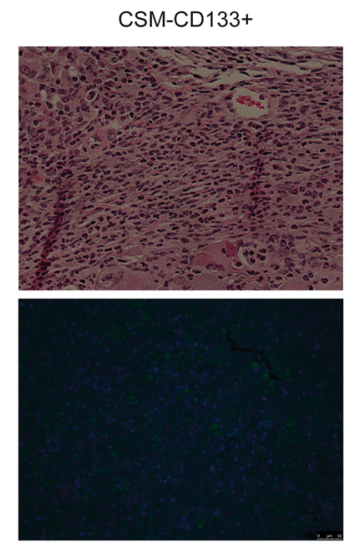

B

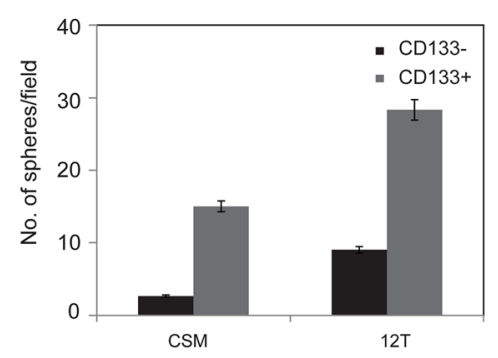

D

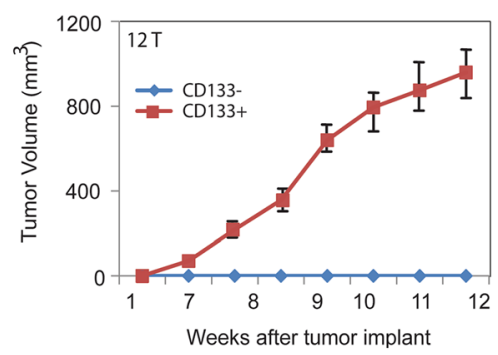

$H \& E$

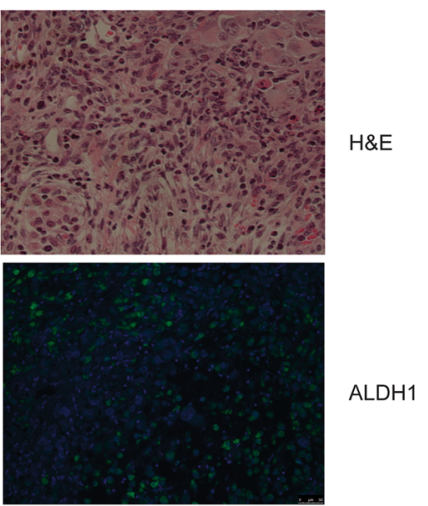

Fig. $3 \mathrm{CD}_{133^{+}}$cells from both CSM and $12 \mathrm{~T}$ cells showed $\mathbf{a}$ increased colony forming ability and $\mathbf{b}$ increased sphere forming ability when compared with CD133- cells. Tumor volume over time from c 500 CSM CD133 ${ }^{+}$cells and d 50012 T CD133 $3^{+}$cells formed tumors in athymic mice while the CD133- cells did not form tumors in either group. e H\&E staining and immunofluorescent staining of ALDH1 of the CSM-CD133 ${ }^{+}$ and 12 T-CD $133^{+}$derived tumors. The error bars represent SEM $\left(n=4\right.$; $\left.^{*}=p<0.05\right)$

showed increased expression for Vimentin compared to CSM cells (Fig. 4d, e). Vimentin expression also correlated with CD133 and ABCG2 protein expression (Fig. 4d). The densitometric quantitation of the protein bands confirmed this observation (Additional file 4: Figure S1).

\section{Tumors derived from $\mathrm{CD} 133^{+}$sorted cells respond to Minnelide}

We have previously shown that Minnelide, a prodrug of triptolide, decreases $\mathrm{CD} 133^{+}$stem cell population in the KPC mouse model of pancreatic cancer [33]. To further establish if tumors derived from $\mathrm{CD} 133^{+}$cells in human cell lines respond to Minnelide, we implanted CD $133^{+}$ cells from CSM and $12 \mathrm{~T}$ into the flanks of 20 athymic nude mice ( $n=10$ for each cohort). Once the tumor volume reached $250 \mathrm{~mm}^{3}$, mice from each cohort were randomized and one cohort was treated with Minnelide. Following 2 weeks of treatment, tumor regression was observed in both CSM-CD $133^{+}$group (Fig. 5a) as well as $12 \mathrm{~T}-\mathrm{CD} 133^{+}$groups, and by the end of the study, tumors from both the group had completely regressed. Tumor weight and tumor volume measurements at the end of the study showed significant reduction in tumor weight (Fig. 5c, d) and tumor volume (Fig. 5e, f).

\section{Discussion}

Accumulating evidence demonstrates that cells from human primary tumors and several cancer cell lines are 


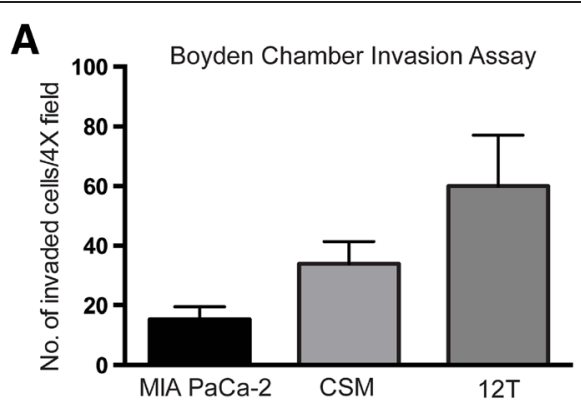

B

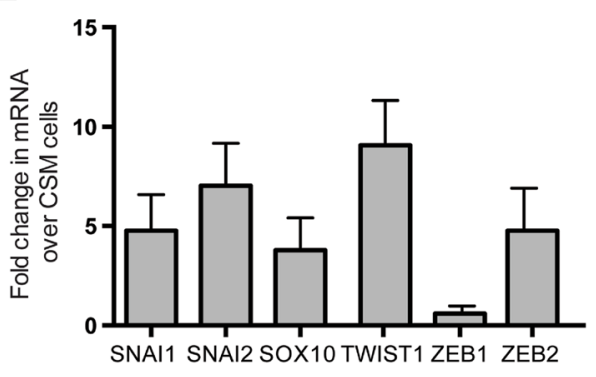

D

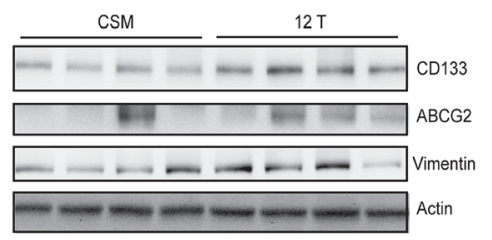

C

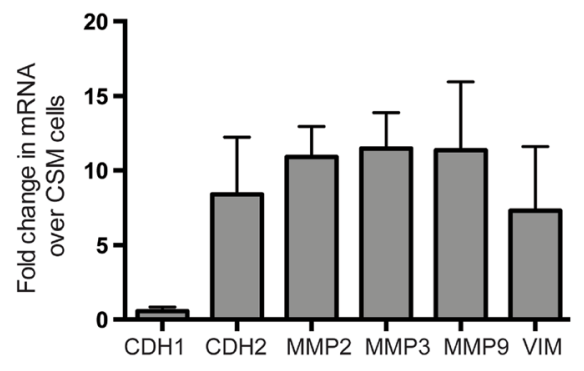

$\mathbf{E}$

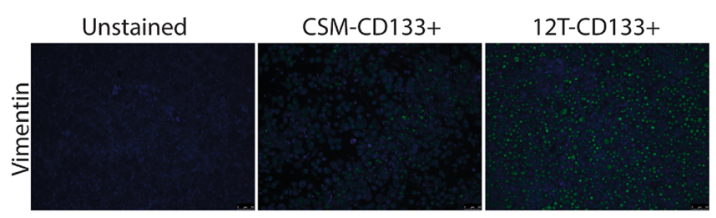

Fig. 4 a Boyden chamber assay showing increased invasion of 12 T cells compared to CSM cells. RNA Expression of transcription factors involved in b EMT and c cytoskeletal re-corganization gene expression in 12 T cells compared to CSM cells. d Protein expression showing positive correlation of CD133, ABCG2 and Vimentin in CSM CD133 ${ }^{+}$and $12 \mathrm{TCD} 133^{+}$tumor bearing animals and e immunofluorescent staining of Vimentin tumor expression. The error bars represent $\operatorname{SEM}\left(n=4 ;{ }^{*}=p<0.05\right)$

heterogeneous and hierarchically organized [34, 35]. A small proportion of tumor cells, designated cancer stem cells (CSCs) or tumor-initiating cells, appear to be associated with the expression of distinct cell surface markers, tumorigenicity and resistance to conventional chemotherapy and radiotherapy [4]. CSCs have properties similar to normal stem cells, such as the ability to self-renew, show drug-efflux, slow cell cycling and differentiate into other cell phenotypes. Moreover, CSCs appear to be correlated with malignance, metastasis, poor prognosis and longterm recurrence $[36,37]$. The heterogeneity of tumors is further demonstrated by the presence of a subset of tumor cells which is able to rapidly efflux lipophilic fluorescent dyes to produce a characteristic profile based on fluorescence-activated flow-cytometric analysis. These cells are referred to as Side Population (SP) cells. SP cells show enrichment for stem-like properties, are able to initiate tumors, and are also resistant to several anti-tumor compounds [38].

It has been suggested that increased exposure to chemotherapeutic compounds resulted in a drug "tolerant" population of cells that show "stemness" properties [39]. Treatment of pancreatic cancer cell lines with low dose of gemcitabine has shown enrichment of "stem-like" cancer cells [40, 41]. Cancer stem cells or tumor initiating cells have been known to become resistant to therapy with continued exposure to chemotherapeutic compounds owing to increased expression of drug transporters like $A B C$ transporters [41, 42]. Interestingly, overexpression of cancer stem cell marker CD133 has been reported to result in up-regulation of the $\mathrm{ABC}$ transporters like ABCG2 [13, 14, 43, 44]. Increased expression of 

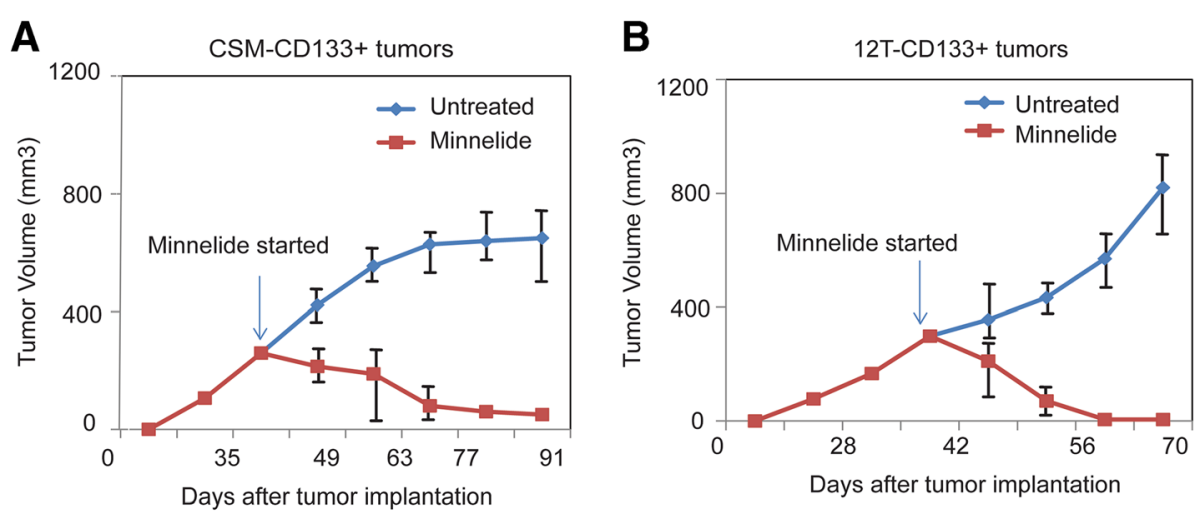

C

D
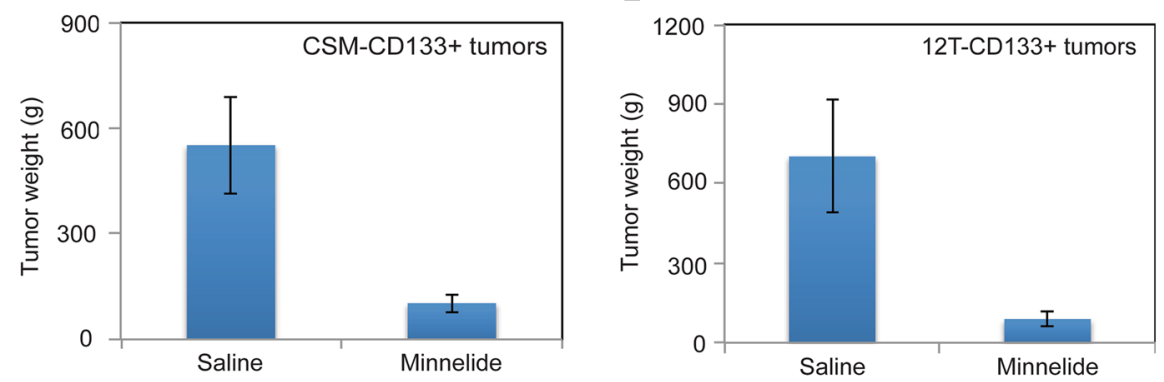

E

F
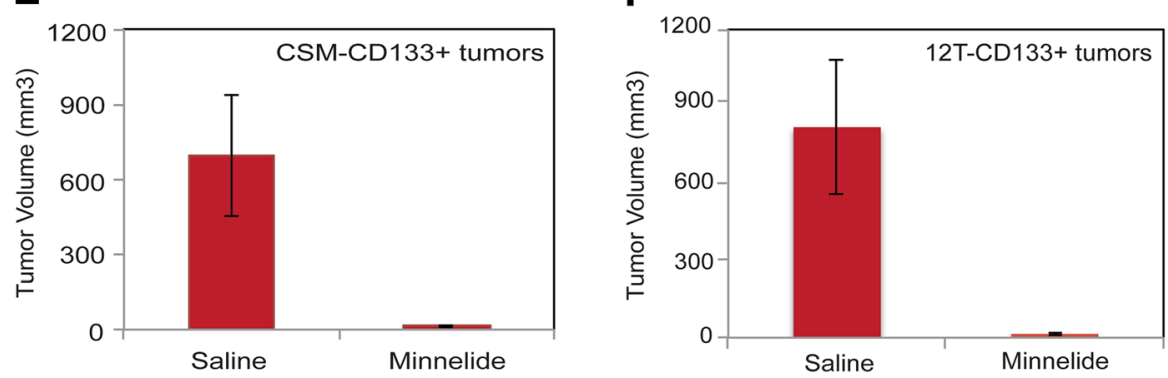

Fig. 5 Tumor progression after implantation of a CSM-CD $133^{+}$cells and $\mathbf{b} 12 \mathrm{~T}-\mathrm{CD} 133^{+}$cells and treatment with Minnelide $(0.42 \mathrm{mg} / \mathrm{kg}$ body weight) in athymic nude mice. Tumor weight of $\mathbf{c}$ CSM-CD $133^{+}$tumors and $\mathbf{d} 12 \mathrm{~T}-\mathrm{CD} 133^{+}$tumors and tumor volume of e CSM-CD $133^{+}$tumors and f $12 \mathrm{~T}$-CD $133^{+}$tumors after treatment with Minnelide. The error bars represent SEM $\left(n=4{ }^{*}=p<0.05\right)$

ABCG2 is also characterized by increased drug efflux property of the cell, increased chemo-resistance, and increased invasion $[14,45,46]$.

In contrast to many conventional drugs, some treatments have been found to induce cell death in tumorinitiating or side population cells with efficacy in pancreatic cancer [32, 47]. Minnelide, the pro-drug of triptolide, a compound derived from a Chinese herb, has shown immense promise in a number of pre-clinical studies. Minnelide is currently undergoing phase I clinical trials at the University of Minnesota- Minneapolis, MN and Mayo Clinic- Scottsdale, AZ, with promising initial responses.

Our previously published study demonstrated that even after treatment with Minnelide is discontinued, the pancreatic tumors do not grow back [47]. This indicated that treatment with Minnelide might be having a deleterious effect on the tumor initiating cells (TICs) in pancreatic cancer. Our earlier results show that treatment with Minnelide is able to significantly reduce the $\mathrm{CD} 133^{+}$population in the spontaneous KPC $\left(\mathrm{Kras}^{\mathrm{G} 12 \mathrm{D}}, \mathrm{Trp} 53^{\mathrm{R} 172 \mathrm{H}}, \mathrm{Pdx}-1-\mathrm{Cre}\right)$ murine model tumors and in human patient tumor derived xenografts [33]. Further, tumors derived from $\mathrm{CD} 133^{+}$population in KPC tumor derived cell line (KPC001) responded to Minnelide as well [33]. These observations were extremely promising. Interestingly, when CD133 was overexpressed in MIA PaCa-2 cells (that have a negligible CD133 expression), we observed increased tumorigenicity in animals [14]. This indicated that CD133 was only just a surface marker that could be used to identify pancreatic TIC, but a surface molecule with complex signaling activity in a cell that resulted in the "stemness" property. When tumors derived from these 
cells was treated with Minnelide, they responded positively as well (Additional file 5: Figure S2).

In the current study, treatment with a very low dose of triptolide $(12.5 \mathrm{nM})$ resulted in selection of a population of cells that were enriched for surface marker CD133 and expression of the drug transporter ABCG2 (Figs. 1 and 2). Additionally, these cells had increased expression of stemness markers and showed tumorigenic potential at very low concentration (Fig. 2). Increased expression of CD133 is reported to correlate with increased invasiveness $[25,48]$. Consistent with this, our studies show that $12 \mathrm{~T}$ cells had increased invasiveness compared to CSM cells (Fig. 3). An analysis of the genes involved in Epithelial-Mesenchymal Transition (EMT) revealed overexpression of genes that down-regulate the epithelial phenotype and up-regulate the mesenchymal phenotype in a cancer cell (Fig. 3). Our studies also revealed that expression of CD133, ABCG2 and invasive markers like Vimentin, positively correlated with each other. Since low dose triptolide treatment resulted in enrichment of $\mathrm{CD}_{133^{+}}$population, we further tested this population for its "stem-like" properties. CD133 cells formed colonies in vitro and also showed sphere formation in vitro. In vivo, the $\mathrm{CD} 133^{+}$population from the triptolide treated $12 \mathrm{~T}$ cells initiated tumors that faster growth than the $\mathrm{CD}_{133^{+}}$population from CSM cells (Fig. 4).

The current study thus established an "in vitro model" for studying the properties associated with cancer stem cells or tumor initiating cells. As seen in cancer stem cells isolated from tumor xenografts, the $12 \mathrm{~T}$ cells showed similar enrichment of surface markers CD24/CD44/ESA and CD133. These cells also showed an increased expression of a number of developmental pathways associated with stemness like the hedgehog and the Notch pathways. Many CSCs are characterized by increased expression of EMT genes and drug transporters like $\mathrm{ABC}$ transporters. The $12 \mathrm{~T}$ cells, which were treated with triptolide for an extended period, showed similar increased expression of EMT along with increased invasiveness. Similarly, these cells also showed an increased expression of ABCG2 and an increased DNA dye efflux (as a measure of increased transporter activity). All these indicated that treatment with a low dose of triptolide in vitro resulted in enrichment of a cancer "stem-like" population was also relatively drug resistant, as reflected by their IC50 values (Additional file 6: Figure S3), and when implanted in animals formed tumors and responded to Minnelide (Fig. 5). Our previously published results indicate that though the $\mathrm{CD}_{133^{+}}$cells were generally resistant to both of the standard chemotherapeutic drugs (gemcitabine, $5 \mathrm{FU}$ and paclitaxel), they were typically responsive to Minnelide [33].

\section{Conclusion}

Recurrence of pancreatic tumors following surgical resection contributes to the poor survival rate of patients diagnosed with this disease. As tumor recurrence is attributed to the presence of cancer stem-like cells in a tumor, the evaluation of any drug against this population is of utmost importance. This study, in addition to our previous work in a syngeneic murine model, provide compelling evidence for Minnelide treatment against pancreatic cancer initiating cells. Minnelide has shown promise in pre-clinical evaluations and is currently undergoing Phase 1 clinical trials.

\section{Methods}

\section{Cells and reagents}

Pancreatic cancer cells MIA PaCa-2, AsPC-1 was obtained from ATCC. S2-013 was a cell line derived from the metastatic SUIT-2 cell line and was a gift from Professor Masato Yamamoto.

MIA PaCa-2 was grown in DMEM-High Glucose with 10 \% FBS, S2-013 was grown and cultured in RPMI with $10 \%$ FBS and AsPC-1 was maintained and cultured in $20 \%$ FBS. All cell growth media included 1X PenicillinStreptomycin.

EGF, FGF, and B12 supplements were obtained from Life technologies. CD133 magnetic beads were obtained from Miltenyi Biotech, CD24, CD44, ESA were purchased from BD Biosciences. Ki-67 antibody was purchased from Thermo Fisher.

\section{Generation of triptolide tolerant cells}

Pancreatic cancer cells MIA PaCa-2, S2-013 and AsPC-1 were treated with $12.5 \mathrm{nM}$ triptolide (in $10 \%$ FBScontaining medium) for 7 days. Following treatments, the viable, triptolide tolerant cells were allowed to recover in a drug free serum containing media for $48 \mathrm{~h}$. The cells were next transferred to a Cancer Stem Cell medium (CSM):F12:DMEM supplemented with EGF, FGF, B27 Supplement along with fungizone and penicillinstreptomycin. These treated cells were referred to as $12 \mathrm{~T}$. In parallel, another batch of cells was grown in full medium without triptolide and was referred to as CSM. $12 \mathrm{~T}$ and CSM cells were used for various cell and animal experiments.

\section{Flow cytometric analysis of isolated samples}

Cells were washed once in PBS and gently scraped into $15 \mathrm{~mL}$ centrifuge tubes, washed in PBS prior to staining and stained with the following directly conjugated monoclonal antibodies in the presence of FcR blocking reagent (Miltenyi Biotech): anti-mouse CD133-PE (Miltenyi Biotech), anti-mouse CD44-FITC (BD Biosciences), ESAAPC (BD Bioscience) and CD24-PE (BD Biosciences, USA). IgG isotype controls corresponding to each directly 
conjugated fluorophore were utilized to identify, quantify, and positively select desired cell populations. All FACS analyses were performed on a BD FACS Canto II (BD Biosciences) using FACS Diva (BD Biosciences) and FlowJo (Tree Star, Ashland, Oregon) software. Debris and cell clusters were excluded during side-scatter and forwardscatter analyses.

\section{RNA and protein analysis}

For protein analysis, the cells were lysed in RIPA buffer (Boston Bioproducts) containing protease inhibitors (Roche) on ice for $15 \mathrm{~min}$. The protein in the cell lysate was quantitated using the BCA protein quantitation kit (Pierce). Equal amount of protein for CSM and $12 \mathrm{~T}$ population was then separated on a SDS PAGE and transferred to nitrocellulose membrane. Expression of different proteins following treatment was studied using western blotting and hybridization.

For transcript analysis, RNA was isolated from this population using Trizol (invitrogen) according to manufacturer's protocol. Real time PCR analysis was performed after synthesizing cDNA (Applied Biosystems) in an ABI7300 instrument (Applied Biosystems).

EMT PCR array (SA Biosciences) was used to study expression level of genes involved in EMT according to manufacturers instruction.

\section{Animal experiment}

All animal experiments were performed according to the University of Minnesota Animal Care Committee guidelines. Sorted cells were washed with serum-free HBSS and suspended in serum free-DMEM/Matrigel mixture (1:1 volume). Either 1000 or 10,000 cells (CSM, $12 \mathrm{~T}$ sorted for $\mathrm{CD}_{133^{+}}$or $\mathrm{CD} 133^{-}$) were injected subcutaneously into the right and left flank of age and gender matched C57BL/6 mice (Jackson Laboratories). 10 mice were used in each group. Tumor were measured weekly and volume was calculated by the formula: $0.52 \times 1 \mathrm{x} \mathrm{w}^{2}$. Tumors were allowed to grow until they reached a volume of $1 \mathrm{~cm}^{3}$, at which the mice were sacrificed and the tumor tissue was harvested and processed for flow cytometry, immunohistochemistry, protein or RNA experiments.

\section{Treatment with Minnelide}

Minnelide treatment was started 5 days after tumor implantation. $0.42 \mathrm{mg}$ Minnelide/ $\mathrm{kg}$ body weight was administered intra-peritoneally QD for 25 days. Tumors were measured as stated above. At the end of study, animals were sacrificed according to the University of Minnesota Animal Care guidelines.

\section{Side population assay/DNA dye efflux assay}

Side population analysis was done according to the protocol of Goodell et al [49]. Briefly, cells (1X $10^{6}$ cells/ $\mathrm{mL}$ ) were incubated in pre-warmed DMEM/5 \% FBS containing freshly added Hoechst $33342(5 \mu \mathrm{g} / \mathrm{mL}$ final concentration) for $90 \mathrm{~min}$ at $37^{\circ} \mathrm{C}$ with intermittent mixing. In some experiments, cells were incubated with the Hoechst dye in the presence of verapamil $(50 \mu \mathrm{mol} /$ L) At the end of incubation, cells were spun down at $4^{\circ}$ $\mathrm{C}$ and resuspended in ice-cold PBS. PI $(2 \mu \mathrm{g} / \mathrm{mL}$ final concentration) was added for $5 \mathrm{~min}$ before fluorescenceactivated cell sorting (FACS) analysis, which allows for the discrimination of dead versus live cells. The Hoechst dye was excited with the UV laser at 351 to $364 \mathrm{~nm}$ and its fluorescence measured with a 515-nm side population filter (Hoechst blue) and a 608 EFLP optical filter (Hoechst red).

\section{Statistical analysis}

Values are expressed as the mean \pm SEM. All in vitro experiments were performed at least three times. Statistical significance of results was calculated using the Student's t-test. Columns represent Mean; bars represent Standard Error $\left(n=4 ;{ }^{*}=p<0.05\right)$.

\section{Additional files}

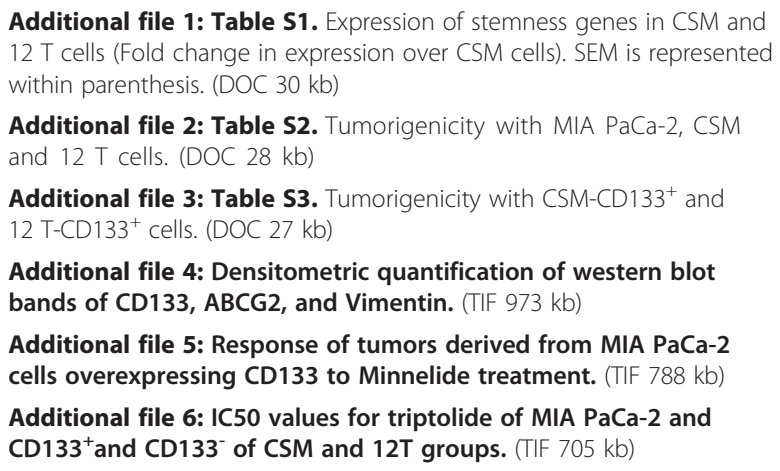

Additional file 3: Table S3. Tumorigenicity with CSM-CD $133^{+}$and $12 \mathrm{~T}^{-C D} 133^{+}$cells. (DOC $27 \mathrm{~kb}$ )

Additional file 4: Densitometric quantification of western blot bands of CD133, ABCG2, and Vimentin. (TIF $973 \mathrm{~kb}$ )

Additional file 5: Response of tumors derived from MIA PaCa-2 cells overexpressing CD133 to Minnelide treatment. (TIF $788 \mathrm{~kb}$ )

Additional file 6: IC50 values for triptolide of MIA PaCa-2 and CD133 ${ }^{+}$and CD133 of CSM and 12 T groups. (TIF $705 \mathrm{~kb}$ )

\section{Abbreviations}

PDAC: Pancreatic Adenocarcinoma; CSC: Cancer Stem Cells; TIC: Tumor initiating cells; CSM: Cancer Stem cell Medium; FACS: Fluorescence Activated Cell Sorting.

\section{Competing interests}

The University of Minnesota has filed a patent for Minnelide ${ }^{\mathrm{TM}}$ (Licensed to Minneamrita Therapeutics LLC, Moline, IL). A.S. has ownership interest (including patents) and is a consultant/advisory board member for Minneamrita Therapeutics. SB is also a consultant with Minneamrita Therapeutics LLC. This relationship has been reviewed and managed by University of Minnesota, in accordance with its conflict of interest policies. The other authors disclosed no potential conflicts of interest.

\section{Authors' contribution}

AN carried out the side population generation, associated experiments and immunohistochemistry. OM carried out the RNA and western blotting on animal and cell samples. VS performed the animal studies. VD, AS, SB conceived of the study, and participated in its design and coordination and helped to draft the manuscript. All authors read and approved the final manuscript. 


\section{Acknowledgement}

The authors would like to thank Dr. Sundaram Ramakrishnan for his valuable inputs in the study. This study was supported by R01-CA170946 (to A.K. Saluja), R01-CA184274 (to S. Banerjee), and Katherine and Robert Goodale foundation; Alice Nomura was supported by T32 DA007097-32.

Received: 25 June 2015 Accepted: 10 November 2015

Published online: 23 November 2015

\section{References}

1. Jemal A, Center MM, DeSantis C, Ward EM. Global patterns of cance incidence and mortality rates and trends. Cancer Epidemiol Biomarkers Prev. 2010:19:1893-907.

2. Griffin JF, Smalley SR, Jewell W, Paradelo JC, Reymond RD, Hassanein RE, et al. Patterns of failure after curative resection of pancreatic carcinoma. Cancer. 1990;66:56-61

3. Cameron JL, Crist DW, Sitzmann JV, Hruban RH, Boitnott JK, Seidler AJ, et al. Factors influencing survival after pancreaticoduodenectomy for pancreatic cancer. Am J Surg. 1991;161:120-4. discussion 124-125.

4. Al-Hajj M, Clarke MF. Self-renewal and solid tumor stem cells. Oncogene 2004;23:7274-82.

5. Bonnet D, Dick JE. Human acute myeloid leukemia is organized as a hierarchy that originates from a primitive hematopoietic cell. Nat Med. 1997:3:730-7.

6. Li C, Heidt DG, Dalerba P, Burant CF, Zhang L, Adsay V, et al. Identification of pancreatic cancer stem cells. Cancer Res. 2007;67:1030-7.

7. Hermann PC, Huber SL, Herrler T, Aicher A, Ellwart JW, Guba M, et al. Distinct populations of cancer stem cells determine tumor growth and metastatic activity in human pancreatic cancer. Cell Stem Cell. 2007;1: 313-23.

8. Immervoll H, Hoem D, Sakariassen PO, Steffensen OJ, Molven A. Expression of the "stem cell marker" CD133 in pancreas and pancreatic ductal adenocarcinomas. BMC Cancer. 2008:8:48.

9. Qu D, Johnson J, Chandrakesan P, Weygant N, May R, Aiello N, et al. Doublecortin-like kinase 1 is elevated serologically in pancreatic ductal adenocarcinoma and widely expressed on circulating tumor cells. PLoS One. 2015;10(2):e0118933. doi: 10.1371/journal.pone.0118933. eCollection 2015

10. Olempska M, Eisenach PA, Ammerpohl O, Ungefroren $H$, Fandrich F, Kalthoff $H$. Detection of tumor stem cell markers in pancreatic carcinoma cell lines. Hepatobiliary Pancreat Dis Int. 2007:6:92-7.

11. Shah AN, Summy JM, Zhang J, Park SI, Parikh NU, Gallick GE. Development and characterization of gemcitabine-resistant pancreatic tumor cells. Ann Surg Oncol. 2007;14:3629-37.

12. Du Z, Qin R, Wei C, Wang M, Shi C, Tian R, et al. Pancreatic cancer cells resistant to chemoradiotherapy rich in "stem-cell-like" tumor cells. Dig Dis Sci. 2011;56:741-50.

13. Angelastro JM, Lame MW. Overexpression of CD133 promotes drug resistance in C6 glioma cells. Mol Cancer Res. 2010;8:1105-15

14. Nomura A, Banerjee S, Chugh R, Dudeja V, Yamamoto M, Vickers SM, et al. CD133 initiates tumors, induces epithelial-mesenchymal transition and increases metastasis in pancreatic cancer. Oncotarget. 2015:6(10):8313-22.

15. Yao J, Cai HH, Wei JS, An Y, Ji ZL, Lu ZP, et al. Side population in the pancreatic cancer cell lines SW1990 and CFPAC-1 is enriched with cancer stem-like cells. Oncol Rep. 2010;23:1375-82.

16. Wang D, Zhu H, Zhu Y, Liu Y, Shen H, Yin R, et al. CD133(+)/CD44(+)/ Oct4(+)/Nestin(+) stem-like cells isolated from Panc-1 cell line may contribute to multi-resistance and metastasis of pancreatic cancer. Acta Histochem. 2013:115:349-56.

17. Goodell MA, Brose K, Paradis G, Conner AS, Mulligan RC. Isolation and functional properties of murine hematopoietic stem cells that are replicating in vivo. J Exp Med. 1996;183:1797-806.

18. Golebiewska A, Bougnaud S, Stieber D, Brons NH, Vallar L, Hertel F, et al. Side population in human glioblastoma is non-tumorigenic and characterizes brain endothelial cells. Brain. 2013;136:1462-75.

19. Golebiewska A, Brons NH, Bjerkvig R, Niclou SP. Critical appraisal of the side population assay in stem cell and cancer stem cell research. Cell Stem Cell. 2011:8:136-47.

20. Kabashima A, Higuchi H, Takaishi H, Matsuzaki Y, Suzuki S, Izumiya M, et al. Side population of pancreatic cancer cells predominates in TGF-beta- mediated epithelial to mesenchymal transition and invasion. Int J Cancer. 2009:124:2771-9.

21. Dou J, Wen P, Hu W, Li Y, Wu Y, Liu C, et al. Identifying tumor stem-like cells in mouse melanoma cell lines by analyzing the characteristics of side population cells. Cell Biol Int. 2009;33:807-15.

22. Abbott BL. ABCG2 (BCRP) expression in normal and malignant hematopoietic cells. Hematol Oncol. 2003;21:115-30.

23. Feuring-Buske M, Hogge DE. Hoechst 33342 efflux identifies a subpopulation of cytogenetically normal CD34(+)CD38(-) progenitor cells from patients with acute myeloid leukemia. Blood. 2001:97:3882-9.

24. Guo D, Xu BL, Zhang XH, Dong MM. Cancer stem-like side population cells in the human nasopharyngeal carcinoma cell line cne-2 possess epithelial mesenchymal transition properties in association with metastasis. Oncol Rep. 2012;28:241-7.

25. Chen S, Song X, Chen Z, Li X, Li M, Liu H, et al. CD133 expression and the prognosis of colorectal cancer: a systematic review and meta-analysis. PLoS One. 2013:8:e56380.

26. Sasaki A, Kamiyama T, Yokoo H, Nakanishi K, Kubota K, Haga H, et al. Cytoplasmic expression of CD133 is an important risk factor for overal survival in hepatocellular carcinoma. Oncol Rep. 2010;24:537-46.

27. Li C, Wu JJ, Hynes M, Dosch J, Sarkar B, Welling TH, et al. c-Met is a marker of pancreatic cancer stem cells and therapeutic target. Gastroenterology. 2011:141:2218-2227.e2215.

28. Jiang R, Li Y, Xu Y, Zhou Y, Pang Y, Shen L, et al. EMT and CSC-like properties mediated by the IKKbeta//kappaBalpha/RelA signal pathway via the transcriptional regulator, Snail, are involved in the arsenite-induced neoplastic transformation of human keratinocytes. Arch Toxicol. 2012;87(6):991-1000.

29. Mirantes C, Espinosa I, Ferrer I, Dolcet X, Prat J, Matias-Guiu X. Epithelial-tomesenchymal transition and stem cells in endometrial cancer. Hum Pathol. 2013; 44(10):1973-81.

30. Aghdassi A, Phillips P, Dudeja V, Dhaulakhandi D, Sharif R, Dawra R, et al Heat shock protein 70 increases tumorigenicity and inhibits apoptosis in pancreatic adenocarcinoma. Cancer Res. 2007;67:616-25.

31. Banerjee S, Thayanithy V, Sangwan V, Mackenzie TN, Saluja AK, Subramanian S. Minnelide reduces tumor burden in preclinical models of osteosarcoma. Cancer Lett. 2013:335:412-20.

32. Chugh R, Sangwan V, Patil SP, Dudeja V, Dawra RK, Banerjee S, et al. A preclinical evaluation of Minnelide as a therapeutic agent against pancreatic cancer. Sci Transl Med. 2012:4:156ra139.

33. Banerjee S, Nomura A, Sangwan V, Chugh R, Dudeja V, Vickers SM, et al. CD133+ tumor initiating cells in a syngenic murine model of pancreatic cancer respond to Minnelide. Clin Cancer Res. 2014;20:2388-99.

34. Cho RW, Clarke MF. Recent advances in cancer stem cells. Curr Opin Genet Dev. 2008;18:48-53.

35. Clevers $\mathrm{H}$. The cancer stem cell: premises, promises and challenges. Nat Med. 2011;17:313-9

36. Monzani E, Facchetti F, Galmozzi E, Corsini E, Benetti A, Cavazzin C, et al. Melanoma contains CD133 and ABCG2 positive cells with enhanced tumourigenic potential. Eur J Cancer. 2007;43:935-46.

37. Adhikari AS, Agarwal N, Iwakuma T. Metastatic potential of tumor-initiating cells in solid tumors. Front Biosci (Landmark Ed). 2011:16:1927-38.

38. Hirschmann-Jax C, Foster AE, Wulf GG, Goodell MA, Brenner MK. A distinct "side population" of cells in human tumor cells: implications for tumor biology and therapy. Cell Cycle. 2005;4:203-5.

39. Hu X, Ghisolfi L, Keates AC, Zhang J, Xiang S, Lee DK, et al. Induction of cancer cell stemness by chemotherapy. Cell Cycle. 2012;11:2691-8.

40. Zhou J, Wang CY, Liu T, Wu B, Zhou F, Xiong JX, et al. Persistence of side population cells with high drug efflux capacity in pancreatic cancer. World J Gastroenterol. 2008;14:925-30.

41. Yin T, Wei H, Gou S, Shi P, Yang Z, Zhao G, et al. Cancer stem-like cells enriched in panc-1 spheres possess increased migration ability and resistance to gemcitabine. Int J Mol Sci. 2011:12:1595-604.

42. Hu G, Li F, Ouyang K, Xie F, Tang X, Wang K, et al. Intrinsic gemcitabine resistance in a novel pancreatic cancer cell line is associated with cancer stem cell-like phenotype. Int J Oncol. 2012;40:798-806.

43. Hueng DY, Sytwu HK, Huang SM, Chang C, Ma HI. Isolation and characterization of tumor stem-like cells from human meningiomas. J Neurooncol. 2011;104:45-53.

44. Warrier S, Pavanram P, Raina D, Arvind M. Study of chemoresistant CD133+ cancer stem cells from human glioblastoma cell line U138MG using multiple assays. Cell Biol Int. 2012;36:1137-43. 
45. Alt R, Wilhelm F, Pelz-Ackermann O, Egger D, Niederwieser D, Cross M. ABCG2 expression is correlated neither to side population nor to hematopoietic progenitor function in human umbilical cord blood Exp Hematol. 2009;37:294-301.

46. Saxena M, Stephens MA, Pathak H, Rangarajan A. Transcription factors that mediate epithelial-mesenchymal transition lead to multidrug resistance by upregulating ABC transporters. Cell Death Dis. 2011;2:e179.

47. Zhang GN, Liang Y, Zhou LJ, Chen SP, Chen G, Zhang TP, et al. Combination of salinomycin and gemcitabine eliminates pancreatic cancer cells. Cancer Lett. 2011;313:137-44

48. Pirozzi G, Tirino V, Camerlingo R, La Rocca A, Martucci N, Scognamiglio G, et al. Prognostic value of cancer stem cells, epithelial-mesenchymal transition and circulating tumor cells in lung cancer. Oncol Rep. 2013;29:1763-8.

49. Goodell MA, McKinney-Freeman S, Camargo FD. Isolation and characterization of side population cells. Methods Mol Biol. 2005;290:343-52.

\section{Submit your next manuscript to BioMed Central and take full advantage of:}

- Convenient online submission

- Thorough peer review

- No space constraints or color figure charges

- Immediate publication on acceptance

- Inclusion in PubMed, CAS, Scopus and Google Scholar

- Research which is freely available for redistribution 